\title{
Article
}

\section{Pressure Effects on a Protein-Lipid Model Membrane}

Kulkarni, Chandrashekhar v., Ces, Oscar, Templer, Richard and Seddon, John

Available at http://clok.uclan.ac.uk/8296/

Kulkarni, Chandrashekhar v. ORCID: 0000-0002-5621-4791, Ces, Oscar, Templer, Richard and Seddon, John (2013) Pressure Effects on a Protein-Lipid Model Membrane. Soft Matter . ISSN 1744-683X

It is advisable to refer to the publisher's version if you intend to cite from the work. http://dx.doi.org/10.1039/C3SM50911G

For more information about UCLan's research in this area go to

http://www.uclan.ac.uk/researchgroups/ and search for < name of research Group>.

For information about Research generally at UCLan please go to http://www.uclan.ac.uk/research/

All outputs in CLoK are protected by Intellectual Property Rights law, including Copyright law. Copyright, IPR and Moral Rights for the works on this site are retained by the individual authors and/or other copyright owners. Terms and conditions for use of this material are defined in the policies page.

\section{CLoK}

Central Lancashire online Knowledge www.clok.uclan.ac.uk

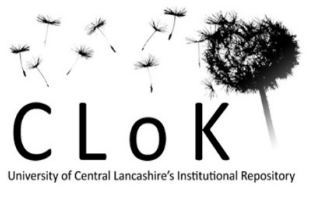




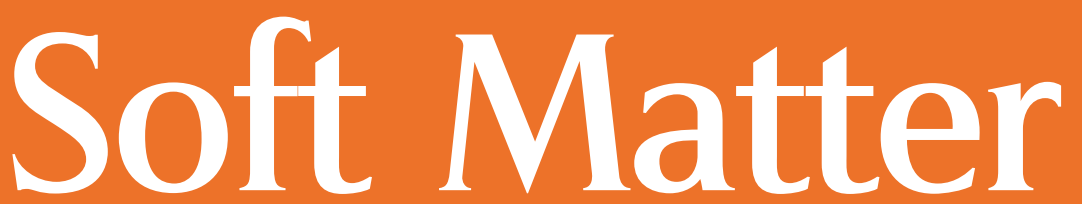

\section{Accepted Manuscript}

This article can be cited before page numbers have been issued, to do this please use: C. V. Kulkarni, O. Ces, R. Templer and J. Seddon, Soft Matter, 2013, DOI: 10.1039/C3SM50911G.

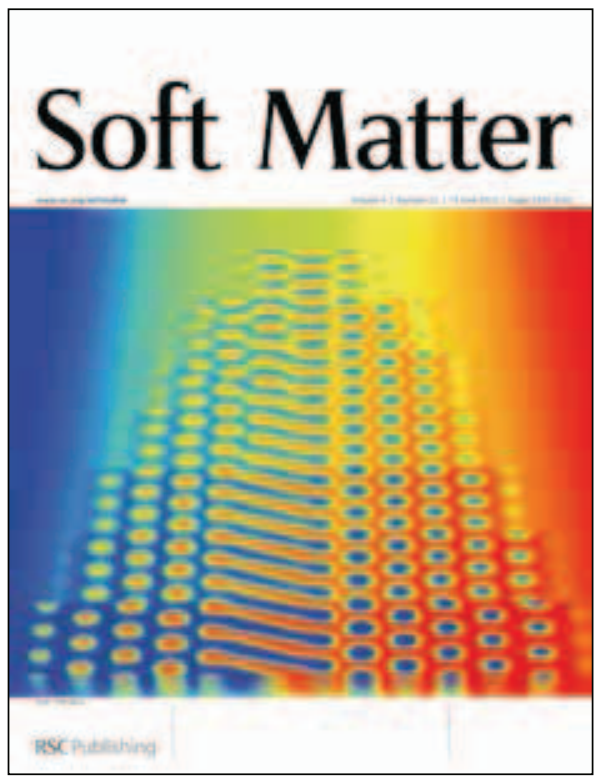

This is an Accepted Manuscript, which has been through the RSC Publishing peer review process and has been accepted for publication.

Accepted Manuscripts are published online shortly after acceptance, which is prior to technical editing, formatting and proof reading. This free service from RSC Publishing allows authors to make their results available to the community, in citable form, before publication of the edited article. This Accepted Manuscript will be replaced by the edited and formatted Advance Article as soon as this is available.

To cite this manuscript please use its permanent Digital Object Identifier $\left.(\mathrm{DO})^{\circledast}\right)$, which is identical for all formats of publication.

More information about Accepted Manuscripts can be found in the

\section{Information for Authors.}

Please note that technical editing may introduce minor changes to the text and/or graphics contained in the manuscript submitted by the author(s) which may alter content, and that the standard Terms \& Conditions and the ethical guidelines that apply to the journal are still applicable. In no event shall the RSC be held responsible for any errors or omissions in these Accepted Manuscript manuscripts or any consequences arising from the use of any information contained in them. 


\title{
Pressure Effects on a Protein-Lipid Model Membrane
}

\author{
Chandrashekhar V. Kulkarni ${ }^{* 1,2}$, Oscar Ces ${ }^{1}$, Richard H. Templer ${ }^{1,3}$ and John
}

M. Seddon*1

${ }^{1}$ Institute of Chemical Biology, Department of Chemistry, Imperial College London, Exhibition Road, London SW7 2AZ, United Kingdom;

${ }^{2}$ Centre for Materials Science, University of Central Lancashire, Preston PRI 2HE, United Kingdom;

${ }^{3}$ Climate-KIC UK, Faculty Building, Imperial College London, Exhibition Road, London SW7 2AZ, United Kingdom.

\begin{abstract}
*Corresponding author e-mails: j.seddon@imperial.ac.uk, cvkulkarni@uclan.ac.uk; Telephones: +44 207594 5797, +44 177289 3491; Fax: +44 1772894981.
\end{abstract}

\begin{abstract}
We attempt to mimic cellular biomembrane structures using a mixture of two important biochemical components - a membrane protein and a lipid, in presence of water. Protein loaded lipid structures were subjected to a wide range of pressures to examine their morphological reorganizations using synchrotron X-ray radiations under stepwise pressure variation and rapid pressure jumps. Here we report the first evidence of a highly swollen gyroid cubic phase under high pressure (gyroid type structures have been seen in ER and Golgi membranes) and the close resemblance of lipid nanostructural behavior with literature studies on compression-decompression of piezophilic biomembranes. These studies are promising for understanding complex
\end{abstract}


biomembrane restructuring and hence functioning, such as how cells cope with extreme conditions of high pressures, and protect their delicate internal organelles.

\section{Introduction}

Lyotropic lipid nanostructures and their hierarchically organized derivatives partly resemble biological configurations like plasma membrane, vesicles, Golgi apparatus, mitochondria and endoplasmic reticulum - ER (both smooth and rough) and also have important implications in their functioning ${ }^{1-8}$. In recent years, lipid based nanostructures are increasingly used as model membranes $^{9-10}$ to study various biological processes for example, membrane fusion ${ }^{11-13}$. Conventional lipid based model membranes primarily involve giant unilamellar vesicles (GUVs), supported lipid bilayers (SLB), or black lipid membranes (BLM) and their derivatives ${ }^{9}$. For better understanding of biological membrane structures and related phenomena, it is essential to study closely analogous lipid based structures under close to physiological conditions. The aforementioned planar bilayer models are not adequate to reflect complex intracellular membranes, for instance, such as the convoluted membrane architecture of endoplasmic reticulum ${ }^{8-9}$.

In the presence of water or aqueous buffers, lipid molecules can self-assemble into a wide range of nanostructures that display $0,1,2$ or 3 dimensional ordered morphologies, corresponding examples being: liposomes (vesicles), flat lamellae (lamellar phase), elongated tube (hexagonal phase) and cubic phases (bicontinuous or micellar), and sometimes disordered architectures (sponge phases) ${ }^{14-}$ 15. Among these, lipid cubic phases are promising models because of their many unique features (Figure 1). Inverse bicontinuous cubic phases are formed from a continuous fluid lipid bilayer 3dimensionally folded in a regular and well-defined morphology, separating two continuous interwoven networks of water channels ${ }^{16-17}$. The cubic phase architecture is based on G (gyroid), D 
(double diamond) and $\mathrm{P}$ (primitive) type of infinite periodic minimal surfaces (IPMS) ${ }^{18}$. The corresponding phases have space groups $I a 3 d, P n 3 m$ and $I m 3 m$, with 3, 4 and 6 water channels meeting at angles of $120^{\circ}, 109.5^{\circ}$, and $90^{\circ}$ respectively. The organized smooth endoplasmic reticulum (OSER) has been shown to exhibit minimal surface topologies of gyroid and diamond types, along with the usual planar lamellae (Figure 1) $)^{8,19}$. Due to the direct structural relevance of lipid cubic phases which 1) have underlying minimal surface geometries and 2) consist of a continuous lipid bilayer that is 3-dimensionally organized, they are more appropriate models for mimicking complex cellular membrane morphologies.

\section{Figure 1}

In general, lipid biomembranes are responsible for structuring and compartmentalization of cells and subcellular organelles. Many important functions are performed cooperatively with membrane proteins, for example, signal transmission, cell differentiation and fusion, protection and insulation of important organs, and biomolecular transport across membranes. In a typical plasma membrane the ratio of lipid to protein is 50:1; as the lipid molecules are very small compared to proteins, membranes thus constitute $\sim 50 \%$ protein by mass. Membrane proteins account for up to a third of the total protein content in the cell; moreover $\sim 70 \%$ of proteins targeted by drugs are membrane $\operatorname{proteins}^{20}$.

In the current work, we have used a model system of two essential cellular components, i.e. a membrane protein and a lipid model membrane. Bacteriorhodopsin contained in the purple membrane (PM) of Halobacterium salinarum was chosen as the membrane protein component because it is very robust under a wide range of physiological and physiochemical conditions and is 
one of the best characterized membrane proteins ${ }^{21-22}$. It doesn't denature until very high pressures in the region of $26 \mathrm{kbar}$, and also does not show significant conformational transitions below 3.7 kbar, making it suitable for the current studies (performed only up to $2.6 \mathrm{kbar})^{23}$. Monoolein (MO) was used as a membrane forming lipid component which is a derivative of $18-\mathrm{C}$ mono-unsaturated fatty acid ${ }^{10}$. The proportion of the latter lipid chain type was found to increase when the culture pressure was increased in case of growth studies of genetically tractable deep-sea bacterium Photobacterium profundum strain $\mathrm{SS}^{24}$. A possible biophysical explanation for this selective preference is that the unsaturated lipids help maintain an optimal level of fluidity under high pressures ('homeoviscous adaptation'), and also keep the membrane in the preferred liquid crystalline phase ('homeophasic adaptive response') in piezophiles ${ }^{24}$. Similarly to the membrane lipids, the membrane proteins are also believed to play an important role in growth under high pressure $^{24}$. To gain insight into this, we have investigated the effect of high pressure on the PMMO system. These type of in vitro studies are particularly advantageous to mimic biomembranes of piezophiles because the latter are disrupted during their transfer from their native deep water environment to the laboratory ${ }^{25}$.

\section{Results}

\section{Compression and decompression of the lipid-membrane protein system}

As well as temperature-water content phase diagrams ${ }^{10}$, pressure-temperature $(\mathrm{p}-\mathrm{T})$ studies on the MO-water system have also been carried out ${ }^{26-29}$. In the excess water region the Pn3m cubic phase was commonly observed, which converts into a lamellar phase beyond pressures of about 2 kbar at $20^{\circ} \mathrm{C}^{27}$. The Pn3m phase has a strong lattice parameter pressure-dependence of $+7.3 \AA / \mathrm{kbar}^{29}$. This characteristic is very common in lipid systems in excess water as the lipid molecules display increased chain splay ${ }^{30}$ with increasing temperature, while the reverse trend is observed when 
hydrostatic pressure is applied. The latter thereby decreases the membrane curvature and thus tends to swell the lyotropic phase, drawing in additional water. There were detectable differences when the PM-MO sample was subjected to high pressure. In this case, the Pn3m to lamellar transition was accompanied by the presence of an Ia3d cubic phase when the pressure was raised from 1 bar (atmospheric pressure) to $2.6 \mathrm{kbar}$ in steps of 200 bar (Figure 2). Both of the cubic phases, Pn3m and Ia3d vanished at $2.6 \mathrm{kbar}$, whereupon a lamellar gel phase $\left(\mathrm{L}_{\beta}\right)$ was solely observed. In the case of PM-MO, the lamellar phase transition occurred at about 500 bar higher pressure than for the pure MO-water system, suggesting that the membrane protein tends to stabilize the curved cubic phases over the flat lamellar phases. The lattice parameters of these cubic phases had an order of magnitude smaller $(\sim 0.75 \AA / \mathrm{kbar})$ pressure dependence as compared to the samples without protein $(7.3 \AA / \mathrm{kbar}$, as mentioned earlier). This observation correlates very well with that revealed independently for pressure effects on proteins and lipids ${ }^{31}$. Lipids were found to be an order of magnitude more pressure sensitive (compressible) than proteins ${ }^{31}$ (further discussed later). In the present case, the membrane protein might be acting as a 'shock absorber', thereby making the overall membrane structure more rigid for pressure perturbations. This could also be the reason why the cubic to lamellar phase boundary was shifted to 500 bar higher pressure.

\section{Figure 2}

Interesting phase behaviour was observed upon systematic pressure reduction from $2.6 \mathrm{kbar}$, (Figure 3). First the $\mathrm{L}_{\beta}$ phase transformed into a fluid lamellar $\left(\mathrm{L}_{\alpha}\right)$ phase, which persisted over the remaining pressure steps down until atmospheric pressure. However at about 300 bar a coexisting highly swollen $P n 3 m$ phase was observed, with an initial lattice parameter of $214 \AA$, 
which shrank by approx $41 \AA$ within one second. At 100 bar, a highly swollen Ia3d phase, with a lattice parameter of $229 \AA$ appeared, which contracted to $168 \AA$ within one second. At atmospheric pressure, the latter further decreased to $159 \AA$, which is in the range of normal Ia3d phase lattice parameters, as reported by Briggs et al. ${ }^{32}$ for $40 \%$ hydrated MO at $20^{\circ} \mathrm{C}$. The results for both upward and downward pressure scans were reproducible even when studied with slightly different pressure steps of 100 bar (plot shown in supplementary information - Figure SI 1). In previous pressure studies of monoelaidin, Im $3 m$ and Pn3m cubic phases were observed in highly swollen states, coexisting with $\mathrm{L}_{\beta}$ and $\mathrm{L}_{\alpha}$ phases respectively ${ }^{37}$. However, to the best of our knowledge the Ia3d phase has never been seen in a highly swollen state during such pressure studies. The observation of a swollen Ia3d phase in the current work gives insight into the effect of membrane protein insertion on MO phase structure. This is an important observation because the ordering of organelle membranes within cells frequently exhibit a gyroid type of morphology. This is the first observation where an Ia3d phase was seen in a highly swollen state for any non-ionic lipid-proteinwater system. (Note also: very large lattice parameter (up to 1,250 ̊) Ia3d phases have been seen in block copolymer systems ${ }^{33}$ ). There have been a small number of reports where addition of a charged lipid led to swelling of the Ia3d cubic phase. Thus, Engblom and co-workers found that addition of an anionic phospholipid to monoolein allowed the Ia3d cubic phase to swell up to a lattice parameter of $275 \AA{ }^{34}$ Similarly, Safinya and co-workers reported that by addition of a cationic phospholipid to monoolein, the Ia3d cubic phase lattice parameter can be swollen to over $200 \AA^{35}{ }^{35}$ These experimental results are in accordance with our recent theoretical model, predicting the possible appearance of an Ia3d phase in the high water region (figure shown in supplementary information Figure SI 2$)^{36}$.

\section{Figure 3}




\section{Pressure jumps}

In case of lyotropic phases it is generally known that the rate of equilibration of the system is faster for pressure changes than temperature changes. ${ }^{10,26}$ Continuous pressure increase or decrease is used to find phase boundaries where transition/coexistence occurs; whereas the pressure jump (pjump) is performed across thus found phase boundaries. Thanks to synchrotron technique one can collect images in extremely small timescales (order of milliseconds) which facilitates understanding of the system far before its final equilibration. By this method the physical interactions occurring at the molecular level (via observation of metastable e.g swollen phases) can be captured in the real time. ${ }^{26}$ In other words, it is possible to understand the way how one phase converts into the other. Such p-jump studies may have direct analogy with the pressure shocks (sudden pressure changes) taking place in the ocean environment. ${ }^{24,25}$ It is therefore worth studying the p-jumps to understand the membrane dynamics that allows cells to tolerate these extreme shocks. Another example of pressure shocks is the process of pasteurization of dairy products involving various lipid nanostructures. Therefore, in addition to static pressure studies (discussed in previous section), some dynamic pressure experiments were performed on the PM-MO system at $20{ }^{\circ} \mathrm{C}$ (Figure 4). In such p-jump studies, the system was first subjected to a pressure above 2 kbar to kill off any coexisting cubic phases, followed by a sudden (within $7 \mathrm{~ms}$ ) decrease in pressure to a low value. The structural changes happening within the first few seconds were detected by capturing a series of images at various time intervals. The following p-jumps were performed on the PM-MO system: 1) 2.2 kbar to 550 bar; 2) 800 bar to 220 bar; 3) 400 bar to 110 bar; and 4) 600 bar to 44 bar. The latter two data sets are discussed below due to their greater relevance to the scope of this paper, and the other two data sets are shown in supplementary information Figure SI 3. 


\section{Figure 4}

During p-jumps, initially the lamellar phase $\left(\mathrm{L}_{\alpha}\right)$ coexisted with one or a maximum of two swollen cubic phases; however, more phases appeared and coexisted as the water content equilibrates throughout the whole system (over $\sim 4$ seconds) (Figure 4). The Roman numerals I, II and III indicate the chronological appearance of the cubic phases. The lattice parameter of the $\mathrm{L}_{\alpha}$ phase decreased only slightly, by $1.4 \AA$ for a p-jump of 400 to 110 bar, and by $3.0 \AA$ during a 600 to 44 bar p-jump. In contrast, the magnitude of the lattice parameter changes was large for the cubic phases. For the 400 to 110 bar p-jump, the $\operatorname{Pn} 3 m(\mathrm{I})$ phase shrank from $201 \AA$ to $142 \AA$, the $P n 3 m$ (II) phase from $164 \AA$ to $122 \AA$ and the $\operatorname{Ia} 3 d(\mathrm{I})$ phase from $213 \AA$ to $182 \AA$. In the case of the 600 to 44 bar pressure-jump, the Pn3m lattice parameter decreased from $195 \AA$ to $145 \AA$, whereas the Ia3d lattice parameter changed from $221 \AA$ to $164 \AA$. Finally, the $\mathrm{L}_{\alpha}$ phase vanished after 10 seconds, to a stable Ia3d phase which had lattice parameters similar to the conventional phase without protein and without pressure cycling. ${ }^{32}$ For the p-jump of 800 to 220 bar, the $\mathrm{L}_{\alpha}$ phase did not vanish and moreover the Ia3d phase appeared only slowly, i.e. after 11 seconds.

More interestingly, when the p-jump was performed from 2.2 kbar down to 550 bar, there was no evidence of an Ia3d phase, and only a swollen Pn3m phase was observed, whose lattice parameter was reduced from $223 \AA$ to $207 \AA$. This means that the swollen Ia3d phase requires decompression to low ( $<550$ bar) pressure conditions. To validate this, we performed two more p-jumps: 2.2 kbar to 1230 bar, and $2.2 \mathrm{kbar}$ to 1630 bar (data not shown). In both of these $\mathrm{p}$-jumps there was no evidence of either swollen or normal cubic phases; instead, the initial lamellar gel phase $\left(\mathrm{L}_{\beta}\right)$ was still observed, which converted into a fluid lamellar $\left(\mathrm{L}_{\alpha}\right)$ phase upon pressure release. 


\section{Discussion}

In previous studies, highly swollen cubic phases were considered to be an intermediate stage during lamellar to cubic transitions. ${ }^{37}$ During rapid alteration of temperature and/or pressure they are presumed to be enclosed by so called 'onion vesicles ${ }^{12}$. The latter are defined as metastable structures where the (multi)lamellar membrane encloses the cubic phase, which can imbibe a large amount of water. ${ }^{12}$ Due to the encapsulation within the lamellar structure the normal interplay of water held by cubic phases is perturbed, causing anomalies in the conventional phase transitions. The observation of more than one $P n 3 m$ and $I a 3 d$ phases during both static and dynamic pressure studies could be attributed to this fact. In other words, the bilayer architecture tries to follow fast equilibration of the released pressure; however the water exchange is relatively slow which is presumed to drive the formation of highly swollen phases while retaining also the lamellar phase. Isothermal decompression studies performed on the membranes of piezophiles closely resemble some of these observations. Electron microscopic examination of these membranes upon decompression from $1.035 \mathrm{kbar}$ showed the formation of intracellular vesicles and smaller membrane fragments, finally leading to cell lysis ${ }^{25}$. Some other structural changes were also seen such as expansion of vacuoles and formation of supramolecular structures such as capsule, fimbriae, pili and flagellae ${ }^{25}$. These morphological changes are attributable to differences in the volumetric compressibility of the involved components, as summarized in Figure 5. The volumetric compressibility of biomembranes and cytoplasm is presumed to differ by $50 \times 10^{-6}$ bar $^{-}$ ${ }^{1,25}$ which would essentially cause biomembranes to expand/compress to a greater extent than cytoplasm. Some independent studies have shown that biomolecular lipid membranes exhibit $\sim 3.5$ times more compressibility $\left(165 \times 10^{-6} \mathrm{bar}^{-1}\right)^{38}$ as compared to water $\left(46 \times 10^{-6} \text { bar }^{-1}\right)^{39}$ under ambient conditions. This may help to explain the formation of biphasic architectures of highly 
swollen cubic within multilamellar onion vesicles. Similarly, an order of magnitude difference between the compressibility of proteins $\left(3-20 \times 10^{-6} \text { bar }^{-1}\right)^{40-41}$ and lipids ${ }^{38}$ could be responsible for differences in the pressure dependence between protein loaded and unloaded lipid cubic phases as discussed above. Red blood cells containing a complex mixture of water, lipids, proteins and various other biochemicals show an intermediate compressibility $\left(33 \times 10^{-6} \text { bar }^{-1}\right)^{42}$ as shown in

\section{Figure 5.}

\section{Figure 5}

Membrane protein reconstitution within lipid phases seems to play a definitive role in their morphological reorganization. As compared to our earlier studies concerning the application of pressure to lyotropic phases and the existence of swollen cubic phases, ${ }^{26,36}$ the present (membrane protein loaded) system was seen to be dominated by Ia3d phases and more importantly the latter were found in highly swollen states. This means that membrane proteins can ease and enhance the formation of gyroid cubic membranes similar to those revealed by in vivo investigations. ${ }^{43}$

\section{Conclusions}

In this work we have studied model membranes under high pressure, providing new insights towards understanding the structural organization of piezophilic membranes. Monoolein, chosen as the lipid component, is a mono-unsaturated fatty acid derivative; such hydrocarbon chains are abundant in the membranes of deep sea water piezophiles. Bacteriorhodopsin, in the form of purple membranes, was selected since it is found naturally in saline water. Notable findings are: 1) a gyroid $(\mathrm{G})$ cubic phase was found in a highly swollen state (lattice parameter $>235 \AA$ ), which was never previously observed in studies involving p-jumps. Subcellular stacked membranes like 
ER (endoplasmic reticulum) and mitochondria under certain conditions are known to form periodically folded structures similar to the G-phase found here. 2) The evolution of structural forms, especially swollen cubic phases presumably located within onion vesicles, is analogous to the morphological changes during decompression of the membranes of piezophiles. 3) A membrane protein affects the barotropic phase behavior of MO, stabilizing more 3-dimensional (cubic) than planar (lamellar) phases. 4) Our model $^{36}$ predicting the possible existence of a Gphase in the high water region appears to be valid. Although the addition of a membrane protein complicates the system, the swollen G-phase was seen reproducibly. For the first time, it was observed for 'static' as well as for 'dynamic' pressure scans. Furthermore, a highly swollen Gphase has not been commonly found in previous studies on lipid-water systems.

\section{Experimental}

\section{Materials}

The lipid component, monoolein (MO) was obtained from Sigma-Aldrich Ltd. (Dorset, UK) and stored at $-20{ }^{\circ} \mathrm{C}$ in amber coloured glass bottles. It was lyophilized before further use. A membrane protein, bacteriorhodopsin (bR) in the form of lyophilized powder of purple membranes (PM) of Halobacterium salinarum was obtained from Munich Innovative Biomaterials GmbH (Munich, Germany). It was stored at $-20{ }^{\circ} \mathrm{C}$ in the dark. The aqueous dispersion was stored at $4{ }^{\circ} \mathrm{C}$. The water used was HPLC-grade, obtained from Sigma-Aldrich Ltd.

\section{Preparation of lipid-protein mixtures}

The lipid-protein samples were prepared by mixing an aqueous solution of PM $(14.67 \mathrm{mg} / \mathrm{ml})$ with MO in 40:60 proportions at room temperature (in the dark). Each mixture contained in $0.5 \mathrm{ml}$ plastic eppendorf tubes was manually mixed using a spatula followed by centrifugation cycles until 
it appeared transparent and/or homogeneous. Samples were subsequently transferred to teflon spacers $(1.5 \mathrm{~mm}$ thick disk, outer diameter $11 \mathrm{~mm}$ with centre hole diameter of $7 \mathrm{~mm})$ and sealed on both sides with X-ray transparent Mylar windows using double sided sticky tape. These sample spacers were directly mounted in the sample chamber of a pressure cell, which has been previously described $^{44}$. The cell permits bidirectional pressure-steps and pressure-jumps of variable amplitude in a time of less than $7 \mathrm{~ms}$ (using pneumatic high-pressure valves situated between the reservoir and the sample chamber). Temperature was controlled at $20 \pm 0.2^{\circ} \mathrm{C}$ via a system of circulating water from a thermostat through the temperature control jacket of the cell. The beamline shutter triggers the electronics controlling the valves so that the p-jump and data acquisition occur simultaneously.

\section{Synchrotron small angle $\mathrm{X}$-ray scattering}

Small angle X-ray scattering (SAXS) studies were performed at the ID02 beamline at the ESRF (European Synchrotron Radiation Facility), Grenoble, France. The beam dimensions used were $200 \mu \mathrm{m}$ vertically and $400 \mu \mathrm{m}$ horizontally. The X-ray wavelength was kept constant at $\lambda=0.07514$ $\mathrm{nm}$ and the sample-to-detector distance was $2.5 \mathrm{~m}$. Measurements were calibrated using silver behenate. Two-dimensional diffraction images were recorded on an image-intensified detector consisting of an X-ray image intensifier (Thomson TTE) coupled to a FreLoN (fast-readout lownoise) charge-coupled device (CCD) developed at the ESRF. The CCD has a frame rate of appr oximately10 frames per second (1024 x 1024 pixels). The identification and analysis of the structural parameters of the observed mesophases was performed using AXcess custom built software based on the IDL (Interactive Data Language) language ${ }^{45}$. To aid the observation of phase transitions, the 3-D stackplots were transformed into intensity plots as shown in this paper. 


\section{Electronic Supplementary Information}

Supplementary information as referred in the text includes additional datasets and a theoretical phase diagram showing re-entrant gyroid phase. See DOI ...

\section{Acknowledgements}

We gratefully acknowledge Prof. Roland Winter, Dortmund University for providing us with his high-pressure X-ray cell. CVK would like to acknowledge a Marie Curie Fellowship (during 20052008) from the European Commission. We would like to thank the EPSRC for Platform grant EP/G00465X, and the European Synchrotron Radiation Facility for the award of beamtime. Support and assistance from Dr. Stephanie Finet and Dr T. Narayanan at beamline ID02 at the ESRF is highly appreciated.

\section{References}

1 Gruner, S. M. Intrinsic Curvature Hypothesis for Biomembrane Lipid Composition: A Role for Nonbilayer Lipids. Proc. Natl. Acad. Sci. USA 82, 3665-3669 (1985).

2 Larsson, K. Cubic Lipid-Water Phases: Structures and Biomembrane Aspects. J. Phys. Chem. 93, 7304-7314 (1989).

3 Fenske, D. B., Monck, M. A., Cullis, P. R. \& Hope, M. J. in Biomembranes: A MultiVolume Treatise Vol. Volume 1 (ed A. G. lee) 1-28 (JAI, 1995).

4 de Kruijff, B. Lipid polymorphism and biomembrane function. Curr. Opin. in Chem. Biol. 1, 564-569 (1997).

5 de Kruijff, B. Biomembranes. Lipids beyond the bilayer. Nature 386, 129-130, (1997).

6 Luzzati, V. Biological significance of lipid polymorphism: the cubic phases. Curr. Opin. in Struct. Biol. 7, 661-668 (1997).

7 Mariani, P., Luzzati, V. \& Delacroix, H. Cubic phases of lipid-containing systems : Structure analysis and biological implications. J. Mol. Biol. 204, 165-189 (1988).

8 Almsherqi, Z. A., Kohlwein, S. D. \& Deng, Y. Cubic membranes: a legend beyond the Flatland of cell membrane organization. J. Cell Biol. 173, 839-844 (2006).

9 Kulkarni, C. V. Lipid crystallization: from self-assembly to hierarchical and biological ordering. Nanoscale 4, 5779-5791 (2012).

10 Kulkarni, C. V., Wachter, W., Iglesias, G. R., Engelskirchen, S. \& Ahualli, S. Monoolein: A Magic Lipid? Physical Chemistry Chemical Physics 13, 3004-3021 (2011).

11 Jahn, R., Lang, T. \& Sudhof, T. C. Membrane Fusion. Cell 112, 519-533 (2003). 
12 Conn, C. E. et al. Dynamics of structural transformations between lamellar and inverse bicontinuous cubic lyotropic phases. Phys. Rev.Letts. 96, 108-102 (2006).

13 Siegel, D. P. \& Kozlov, M. M. The Gaussian Curvature Elastic Modulus of NMonomethylated Dioleoylphosphatidylethanolamine: Relevance to Membrane Fusion and Lipid Phase Behavior. Biophys. J. 87, 366-374 (2004).

14 Hyde, S. T. in Handbook of Applied Surface and Colloid Chemistry (ed Krister Holmberg) Ch. 16, 299-332 (John Wiley \& Sons, Ltd., 2001).

15 Seddon, J. M. \& Templer, R. H. in Handbook of Biological Physics Vol. Volume 1 Ch. 3, 97-160 (Elsevier Science B.V. , 1995).

16 Hyde, S. T. Bicontinuous structures in lyotropic liquid crystals and crystalline hyperbolic surfaces. Curr. Opin. in Solid State Mater. Sci. 1, 653-662 (1996).

17 Seddon, J. M. \& Templer, R. H. Cubic Phases of Self-assembled Amphiphilic Aggregates. Philos. Trans Roy. Soc. A. 344, 377-401 (1993).

18 Larsson, K. Periodic minimal surface structures of cubic phases formed by lipids and surfactants. J.Coll. Interface Sci. 113, 299-300 (1986).

19 Snapp, E. L. et al. Formation of stacked ER cisternae by low affinity protein interactions. J.Cell Biol. 163, 257-269 (2003).

20 Kulkarni, C. V. in Advances in Planar Lipid Bilayers and Liposomes Vol. 12 (ed Ales Iglic) Ch. 9, 237-272 (Academic Press, 2010).

21 van Noort, J. Unraveling bacteriorhodopsin. Biophys. J. 88, 763-764 (2005).

22 Lanyi, J. K. Bacteriorhodopsin. Ann. Rev.Physiol. 66, 665-688 (2004).

23 Barnett, S. M., Edwards, C. M., Butler, I. S. \& Levin, I. W. Pressure-Induced Transmembrane $\alpha_{I^{-}}$to $\alpha_{\mathrm{I}^{-}}$Helical Conversion in Bacteriorhodopsin: An Infrared Spectroscopic Study. J. Phys. Chem. B 101, 9421-9424 (1997).

24 Bartlett, D. H. Pressure effects on in vivo microbial processes. Biochim.Biophys. Acta 1595, 367-381 (2002).

25 Chastain, R. A. \& Yayanos, A. A. Ultrastructural changes in an obligately barophilic marine bacterium after decompression. Appl. Environ. Microbiol. 57, 1489-1497 (1991).

26 Conn, C. E. et al. A pressure-jump time-resolved X-ray diffraction study of cubic-cubic transition kinetics in monoolein. Langmuir 24, 2331-2340 (2008).

27 Pisani, M., Bernstorff, S., Ferrero, C. \& Mariani, P. Pressure Induced Cubic-to-Cubic Phase Transition in Monoolein Hydrated System. J.Phys. Chem. B 105 (2001).

28 Mariani, P. et al. Effects of hydrostatic pressure on the monoolein-water system: An estimate of the energy function of the inverted Ia3d cubic phase. Phys. Rev. E 54, 5840 (1996).

29 Czeslik, C., Winter, R., Rapp, G. \& Bartels, K. Temperature- and pressure-dependent phase behavior of monoacylglycerides monoolein and monoelaidin. Biophys. J. 68, 1423-1429 (1995).

30 Kulkarni, C. V. et al. Engineering Bicontinuous Cubic Structures at the Nanoscale- the Role of Chain Splay. Soft Matter 6, 3191-3194 (2010).

31 Weber, G. \& Drickamer, H. G. The effect of high pressure upon proteins and other biomolecules. Q. Rev. Biophys. 16, 89-112 (1983).

32 Briggs, J., Chung, H. \& Caffrey, M. The temperature-composition phase diagram and mesophase structure characterization of the monoolein/water system. J. Phys. II France $\mathbf{6}$, 723-751 (1996).

33 Vukovic, I. et al. Double Gyroid Network Morphology in Supramolecular Diblock Copolymer Complexes. Macromolecules 45, 3503-3512(2012). 
34 Engblom, J., Miezis, Y., Nylander, T., Razumas, V. \& Larsson, K. in Progress in Colloid and Polymer Science : Surface and Colloid Science Vol. 116 9-15 (Springer-Verlag, 2000).

35 Leal, C., Bouxsein, N. F., Ewert, K. K. \& Safinya, C. R. Highly Efficient Gene Silencing Activity of siRNA Embedded in a Nanostructured Gyroid Cubic Lipid Matrix. J. Am. Chem. Soc. 132, 16841-16847 (2010).

36 Shearman, G. C., Ces, O. \& Templer, R. H. Towards an understanding of phase transitions between inverse bicontinuous cubic lyotropic liquid crystalline phases. Soft Matter 6, 256262(2010).

37 Shearman, G. C. et al. Calculations of and Evidence for Chain Packing Stress in Inverse Lyotropic Bicontinuous Cubic Phases. Langmuir 23, 7276-7285 (2007).

38 Carius, W. The compressibility of bimolecular lipid membranes. Biophys. Struct. Mechanism 6, 90-90 (1980).

39 Fine, R. A. \& Millero, F. J. Compressibility of water as a function of temperature and pressure. J. Chem. Phys.59, 5529-5536 (1973).

40 Gekko, K. \& Noguchi, H. Compressibility of globular proteins in water at 25.degree.C. $J$. Phys. Chem. 83, 2706-2714(1979).

41 Hianik, T., Buckin, V. A. \& Piknova, B. Can a single bacteriorhodopsin molecule change the structural state of one liposome? Gen. Physiol.Biophys. 13, 493-501 (1994).

42 Hartono, D. et al. On-chip measurements of cell compressibility via acoustic radiation. $L a b$ on a chip 11, $4072-4080$ (2011).

43 Almsherqi, Z., Margadant, F. \& Deng, Y. in Advances in Planar Lipid Bilayers and Liposomes Vol. 12 Ch. 4, 79-99 (Elsevier, 2010).

44 Woenckhaus, J., Kohling, R., Winter, R., Thiyagarajan, P. \& Finet, S. High pressure-jump apparatus for kinetic studies of protein folding reactions using the small-angle synchrotron x-ray scattering technique. Rev.Sci. Instrum. 71, 3895-3899 (2000).

45 Seddon, J. et al. Pressure-jump X-ray studies of liquid crystal transitions in lipids. Philos. Trans. Roy. Soc. A 364, 2635-2655 (2006).

\section{Figure Captions}

Figure 1: Resemblance between cellular biomembranes and lipid cubic phases ${ }^{8,19}$ : a) Various types of biomembrane foldings occurring in the organized smooth endoplasmic reticulum (OSER): Continuity between lamellar (L) and sinusoidal (S) type of ER is clearly visible analogous to lamellar and cubic lipid phases; in the vicinity the clusters of mitochondria (M) can be seen clearly similar to lipid vesicular structures. b) Theoretically superimposed projections of balanced (2parallel surfaces) elucidate that ER can exhibit regular gyroid (left) and double diamond (right) like architecture. c) Mathematically ordered minimal surfaces which form the basis for 3- 
dimensional lipid cubic phases shown in d). Images in a) and b) were obtained from references ${ }^{19}$ and ${ }^{8}$ respectively and those in c) and d) were modified from reference ${ }^{20}$.

Figure 2: Upward pressure scan in 200 bar steps of PM-MO in excess water: a) A stacked plot of diffraction pattern in reciprocal spacing $(\mathrm{S})$ versus pressure indicates the phase transition from an initial Pn3m phase to a lamellar gel phase $\left(\mathrm{L}_{\beta}\right)$ above $2.5 \mathrm{kbar}$. The appearance of the Ia3d phase is a consequence of the presence of the membrane protein within the cubic phase. The numbers in green specify the corresponding peak-fit indices for Pn3m, Ia3d and $\mathrm{L}_{\beta}$ phases. b) The lattice parameters of the cubic phases slightly $(\sim 2 \AA)$ increase over the 2.5 kbar pressure range. The inset shows the increasing lattice parameter for the Pn3m phase with pressure. Block arrows indicate the time course direction of the experiment.

Figure 3: Highly swollen cubic phases during the downward pressure scan: a) The diffraction pattern scan shows the sharp phase transition from $\mathrm{L}_{\beta}$ (gel) to $\mathrm{L}_{\alpha}$ (fluid lamellar phase), which is followed by the appearance of highly swollen cubic phases Pn3m and Ia3d that subsequently evolve into a classical Ia3d cubic phase. The pressure was decreased from 2.6 kbar in 200 bar steps down to 300 bar, at which point time- resolved (designated by $\mathrm{f}(\mathrm{t}) \mathrm{I}$ ) studies were performed to capture the time evolution of the swollen phases. Similarly, time-resolved studies $\mathrm{f}(\mathrm{t}) \mathrm{II}$ and $\mathrm{f}(\mathrm{t}) \mathrm{III}$ were performed to capture the corresponding kinetics at 100 bar and at atmospheric pressure. The numbers in green specify the corresponding peak-fit indices for the $\mathrm{L}_{\alpha}$ phase. b) The lattice parameters of all phases except $\mathrm{L}_{\beta}$ were found to decrease particularly rapidly during coexistence with other phases. The time-resolved change in the lattice parameters of the swollen Pn3m, the swollen Ia3d and the normal (classical) Ia3d phase are shown in c), d) and e) respectively. 
Figure 4: Pressure jumps showing swollen cubic phases: p-jumps from 400 bar a) \& b), and 600 bar c) \& d) to atmospheric pressure. The asterisked values in brackets indicate the actual pressure values attained when the jumps were complete. At the initial high pressure, the fluid lamellar $\mathrm{L}_{\alpha}$ phase is observed with a d-spacing of approx $51 \AA$, while a mixture of swollen phases was seen as the system relaxes over $\sim 4$ seconds towards atmospheric pressure. The shaded area indicates multiple phase coexistence. The time-resolved behaviour of disappearing and evolving phases can be seen from the peak positions in a) and c) and the lattice parameters as a function of time in b) and d). Due to less intense and broad peaks it was hard to determine lattice parameters of some swollen phases, for instance, Ia3d(III), which is thus not plotted. Note the appearance and coexistence of cubic phases $\operatorname{Pn} 3 m$ (I), Pn3m (II), Ia3d (I), Ia3d (II), Ia3d (III) is indicated by high intensity lines on the left and by lattice parameter change on the right.

Figure 5: Comparison of compressibilities: Compressibility values of various cellular components under ambient conditions obtained from various references as follows: water - ${ }^{39}$, noctane $-{ }^{38}$, biomolecular lipid membranes $-{ }^{38}$, proteins $-{ }^{40-41}$ and red blood cells $-{ }^{42}$. The n-Octane value is shown as an example of a typical hydrophobic molecule. 


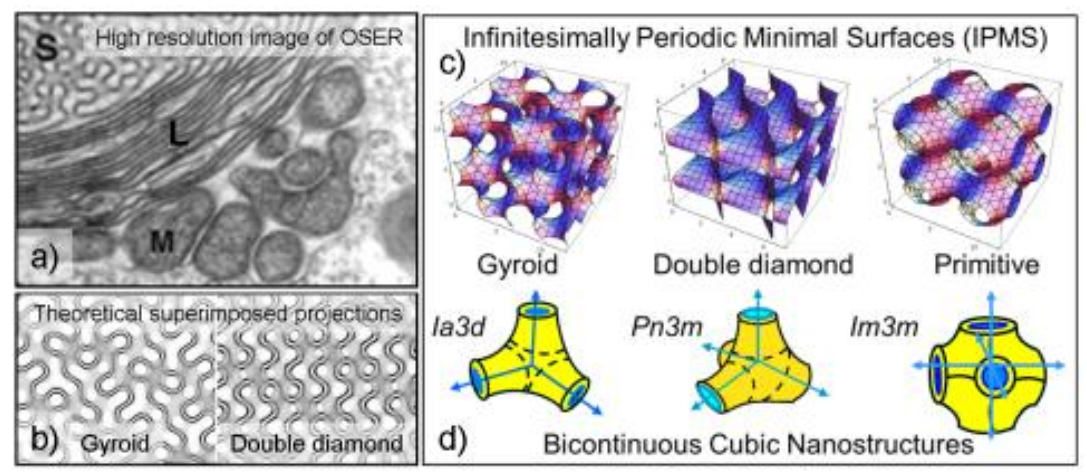

Figure 1

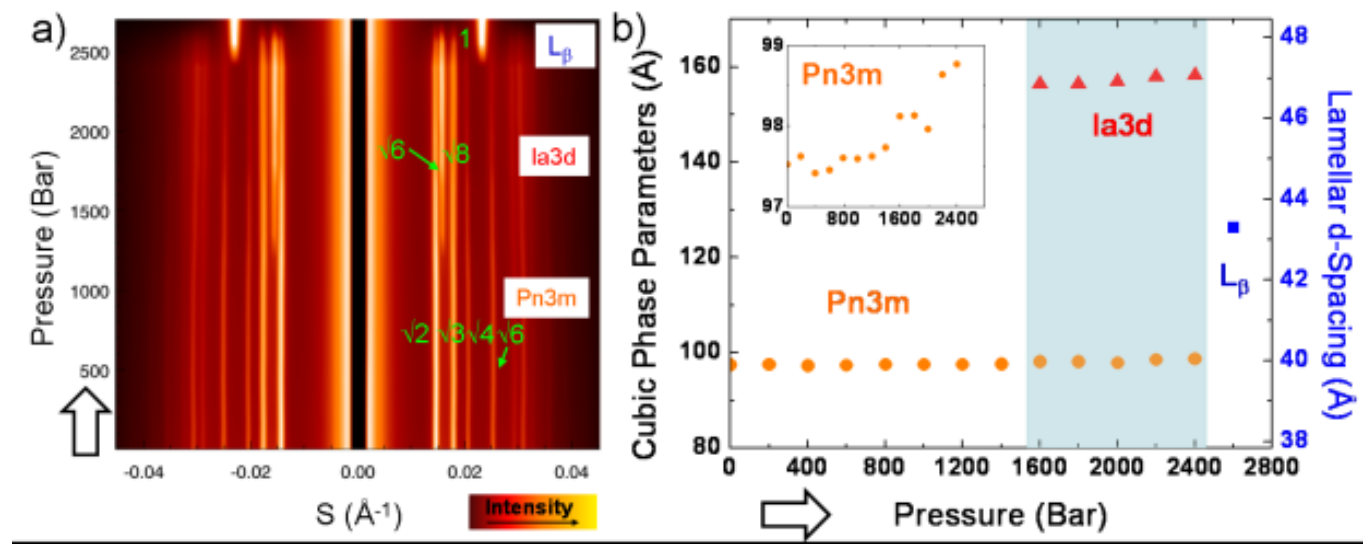

Figure 2
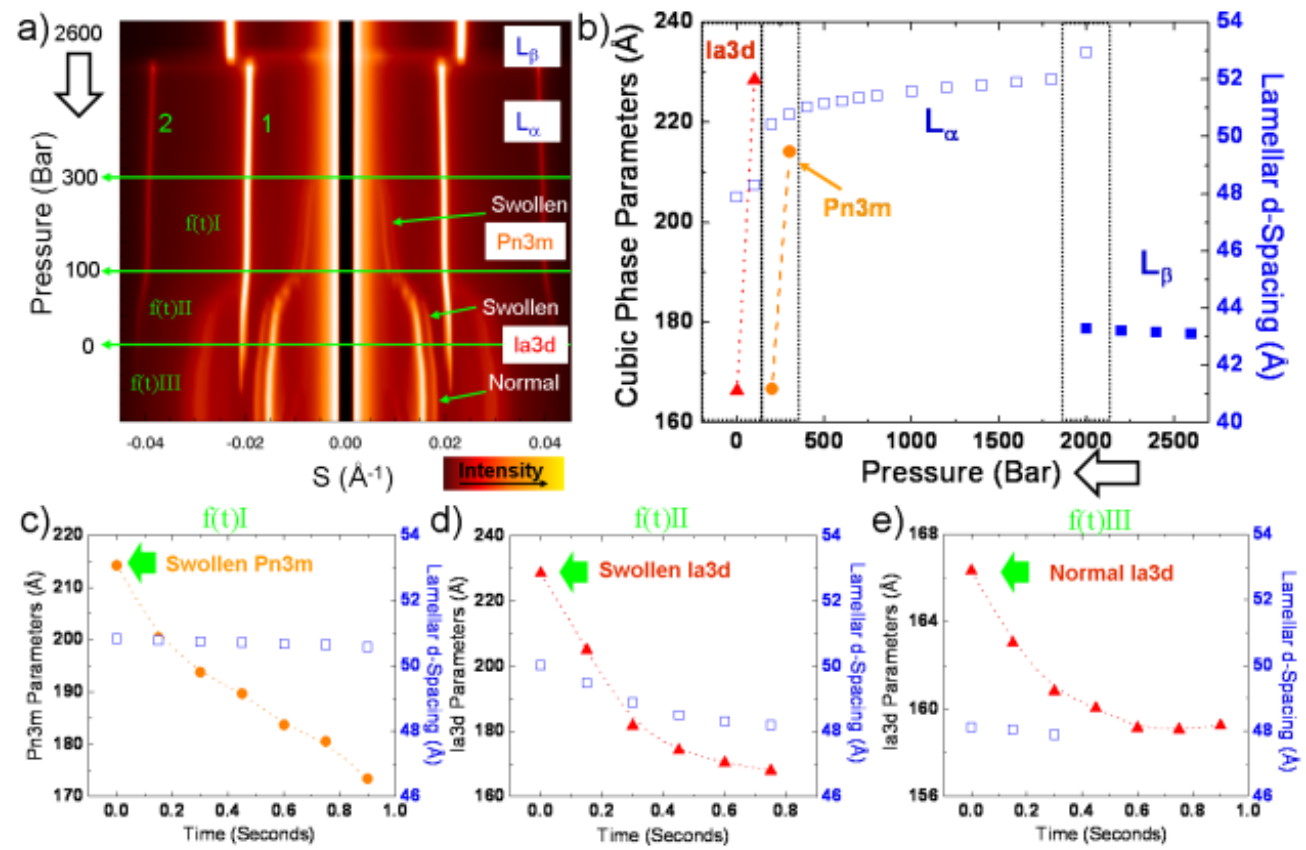

${ }_{54}$ d) ${ }_{240}$

Figure 3

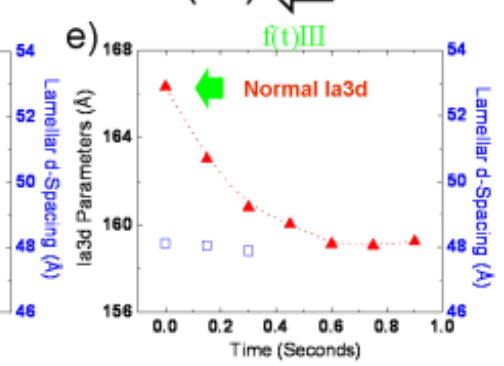



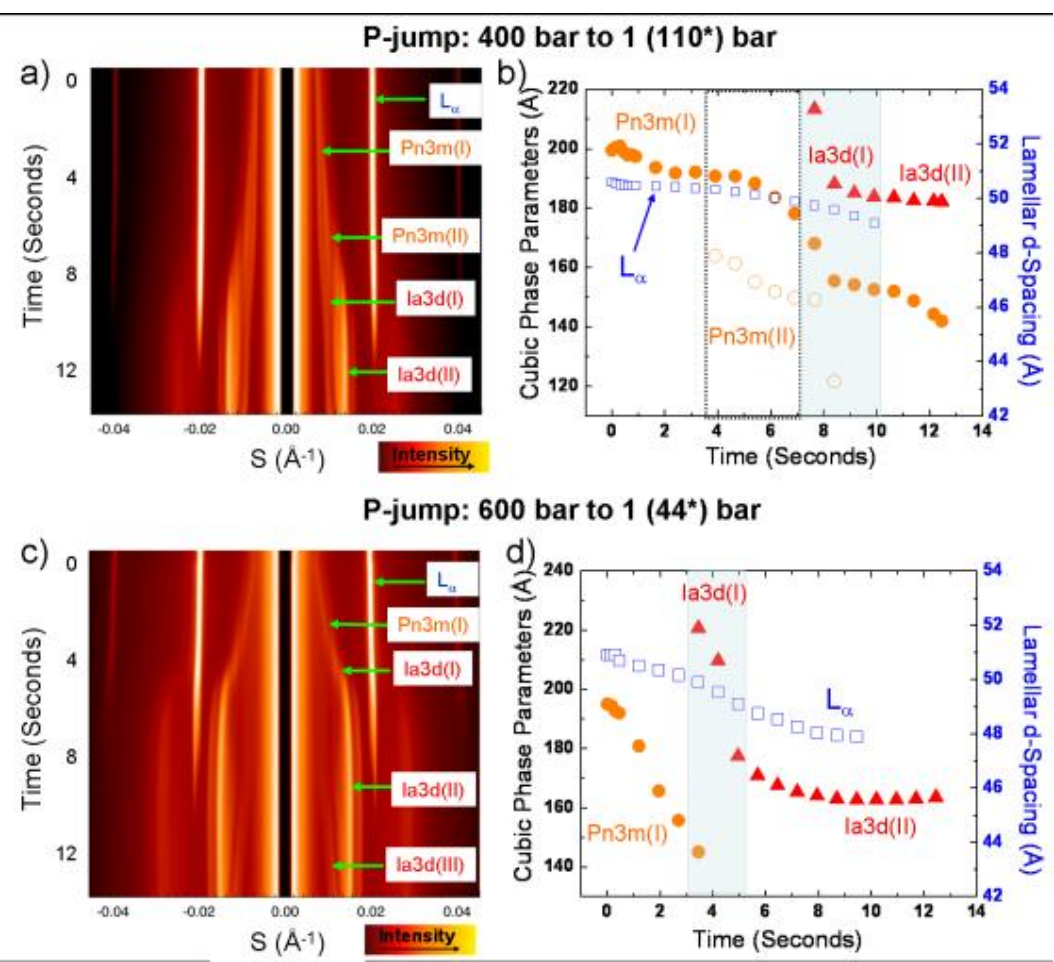

Figure 4

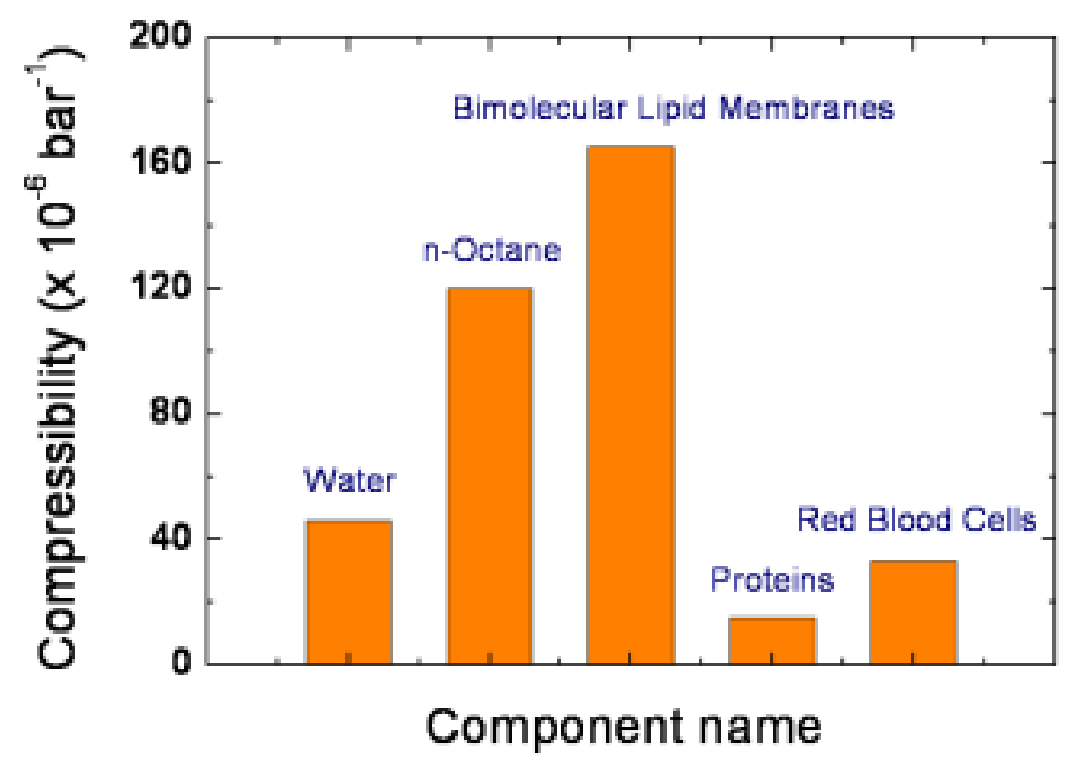

Figure 5 